\title{
Endosonics: Revolutionizing Endodontics
}

\author{
Dr. Pradnya V. Bansode ${ }^{1}$, Dr. Seema D. Pathak ${ }^{2}$, Dr. M. B. Wavdhane ${ }^{3}$, \\ Dr. Shirish B. Khedgikar ${ }^{4}$, Dr. Anjali Pandey ${ }^{5}$ \\ ${ }^{1}$ Professor and Head Of Department, (Department Of Conservative Dentistry and Endodontics, \\ GDC \& Hospital, Aurangabad/MUHS, India). \\ ${ }^{2}$ Associate Professor, (Department Of Conservative Dentistry and Endodontics, GDC \& Hospital, \\ Aurangabad/ MUHS, India) \\ ${ }^{3}$ Associate Professor, (Department Of Conservative Dentistry and Endodontics, GDC \& Hospital, \\ Aurangabad/ MUHS, India) \\ ${ }^{4}$ Lecturer, (Department Of Conservative Dentistry and Endodontics, GDC \& Hospital, \\ Aurangabad/MUHS, India) \\ ${ }^{5}$ Second Year Post-Graduate Student, (Department Of Conservative Dentistry and Endodontics, \\ GDC \& Hospital, Aurangabad/ MUHS, India).
}

\begin{abstract}
During the past few decades endodontic treatment has benefited from the development of new techniques and equipment, which have improved outcome and predictability. Important attributes such as the operating microscope and ultrasonics (US) have found indispensable applications in a number of dental procedures in periodontology, to a much lesser extent in restorative dentistry, while being very prominently used in endodontics. US in endodontics has enhanced the quality of treatment and represents an important adjunct in the treatment of difficult cases. Since its introduction, US has become increasingly more useful in applications such as gaining access to canal openings, cleaning and shaping, obturation of root canals, removal of intracanal materials and obstructions, and endodontic surgery. This comprehensive review of the literature aims at presenting the numerous uses of US in clinical endodontics and emphasizes the broad applications in a modern-day endodontic practice.
\end{abstract}

Keywords: Endodontics, Endosonics, Piezoeclectric, troughing, Ultrasonics.

\section{Introduction}

The use of ultrasonics (US) or ultrasonic instrumentation was first introduced in dentistry for cavity preparations using an abrasive slurry. But uptil 1957 it was basically used for removal of calculus and stains from teeth. The concept of using US in endodontics was first introduced by Richman [1]. However, it was not until Martin et al demonstrated the ability of ultrasonically activated K-type files to cut dentin that this application found in ommon use in the preparation of root canals before obturation. The term endosonics $[2,3]$ was coined by Martin and Cunningham and was defined as the ultrasonic and synergistic system of root canal instrumentation and disinfection.

Ultrasound is sound energy with a frequency above the range of human hearing which is $20 \mathrm{kHz}$. The range of frequencies employed in the original ultrasonic units was between 25 and $40 \mathrm{kHz}$ [4]. Subsequently the so-called low-frequency ultrasonic handpieces operating from 1 to $8 \mathrm{kHz}$ were developed, which produce lower shear stresses, thus causing less alteration to the tooth surface. There are two basic methods of producing ultrasound. The first is magnetostriction, in which a stack of magnetostrictive metal strip in a handpiece is subjected to a standing and alternating magnetic field, as a result of which vibrations are produced. The second method is based on the piezoelectric principle, in which deformation of this crystal is converted into mechanical oscillation without producing heat [4].

Piezoelectric units have some advantages compared with earlier magnetostrictive units because they offer more cycles per second, 40 versus $24 \mathrm{kHz}$. The tips of these units work in a linear, back-and-forth, "pistonlike" motion, which is ideal for endodontics. This applies in particular when "troughing" for hidden canals or when removing posts and separated instruments. In addition, this motion is ideal in surgical endodontics when creating a preparation for a retrograde filling. A magnetostrictive unit, on the other hand, creates more of a figure eight (elliptical) motion, which is not ideal for either surgical or nonsurgical endodontic use. The magnetostrictive units also have the disadvantage that the stack generates heat, that requires adequate cooling. 


\section{Applications of US in Endodontics}

1. Access refinement, finding calcified canals, and removal of attached pulp stones.

2. Removal of intracanal obstructions (separated instruments, root canal posts, silver points, and fractured posts).

3. Increased action of irrigating solutions.

4. Ultrasonic condensation of gutta-percha.

5. Placement of mineral trioxide aggregate (MTA).

6. Surgical endodontics: Root-end cavity preparation and refinement and placement of root-end obturation material.

7. Root canal preparation.

\section{Access refinement, finding calcified canals, and removal of attached pulp stones}

One of the challenges in endodontics is to locate canals, particularly in cases in which the orifice has become occluded by secondary dentin or calcified dentin secondary to the placement of restorative materials or pulpotomies. With every access preparation in a calcified tooth, there is the risk of perforating the root or, when incorrectly performed, of complicating each subsequent procedure. A lack of a straight-line access is arguably the leading cause of separation, perforation, and the inability to negotiate files to the radiographic terminus.

The introduction of the microscope, access burs, and US has greatly reduced these risks. Microscopic visualization and ultrasonic instruments are a safe and effective combination to achieve optimal results. In difficult-to-treat teeth, US has proven to be useful for access preparation, not only for finding canals, but also for reducing the time and the predictability of the treatment. There are numerous variations of rotary access burs available; however, one of the more important advantages of ultrasonic tips is that they do not rotate, thus enhancing safety and control, while maintaining a high cutting efficiency. This is especially important when the risk of perforation is high. The visual access and superior control that ultrasonic cutting tips provide during access procedures make them a most convenient tool, especially when treating difficult molars. When locating the MB2 canals in upper molars [5], US is an excellent means for the removal of secondary dentin on the mesial wall. When searching for hidden canals, one should remember that secondary dentin is generally whitish or opaque, whereas the floor of the pulp chamber is darker and gray in appearance. US works well when breaking through the calcification that covers the canal orifice. A troughing tip is a good choice for this task. For these applications, bigger tips with a limited diamond coated extension should be used during the initial phase of removing calcification, interferences, materials, and secondary dentin, as they offer maximum cutting efficiency and enhance control while working in the pulp chamber. The subsequent phase of finding canal orifices should be carried out with thinner and longer tips that facilitate working in deeper areas while maintaining clear vision.

\section{Removal Of Intracanal Obstructions}

Clinicians are frequently challenged by endodontically treated teeth that have obstructions such as hard impenetrable pastes, separated instruments, silver points, or posts in their roots. If endodontic treatment has failed, these obstructions need to be removed to perform nonsurgical retreatment. Ultrasonic energy has proven effective as an adjunct in the removal of silver points, fractured instruments, and cemented posts.

\section{Separated Instruments}

Management of a broken instrument requires an orthograde or a surgical approach. The three orthograde approaches [6] are (a) attempt to remove the instrument; (b) attempt to bypass the instrument; and (c) prepare and obturate to the fractured segment. US has proved to be useful in removal of separated instrument most of the times, without excessive removal of tooth structure.

\section{Root Canal Posts}

US has provided clinicians with a useful adjunct to facilitate post removal with minimal loss of tooth structure and root damage. Many studies have focused on the removal of metallic posts; however, retreatment of fiber-reinforced composite posts cemented with adhesive systems presents a new challenge in cases in which endodontic treatment has failed. Different bur kits have been proposed to remove fiber posts; however, the preservation of maximum root structure requires the use of specific ultrasonic tips and adequate magnification. The disruption of the composite structure through the action of ultrasonic vibration seems to be the most effective technique in fiber post removal. Esthetic white posts are more difficult to remove because their color matches that of dentin, whereas the black carbon fiber posts clearly contrast to dentin. Removal is done in a dry field using a continuous stream of air with direct vision of the ultrasonic tip and the coronal portion of the post, alternated by air and water spray to clean the remnants of fibers and dentin. If the ultrasonic tip leaves behind gray streaks, it is a clear indication that resin composite or resin composite cement is still present. 
The need of consuming fiber posts is based on the fact that the viscoelastic nature of composite resin dampens vibrations and adsorbs energy [7]. Conductance of vibration forces within a post is proportional to the square root of the modulus of elasticity of the post material. Therefore, a fiber-reinforced composite post with a significantly lower modulus of elasticity than stainless steel or titanium conducts vibration less efficiently. Combining the low modulus of elasticity of post materials with composite resin cements causes a change in the effectiveness of US as an aid in post removal. Resin cements are not friable and do not tend to produce microfractures due to ultrasonic vibration. It was suggested that the absence of a water spray seems to increase the action of US when applied to posts cemented with resin cements, possibly because of the increase in heat. This is helpful information, as it has been observed that the capacity of adhesion of a resin cement, and consequently mechanical retention, gradually reduces with the number of thermal cycles.

\section{Silver Points and Fractured Metallic Posts}

The traditional clinical procedure to remove root canal posts or silver points fractured at the orifice consists of exposing the coronal part of the obstacle by cutting an estimated $2.0-\mathrm{mm}$ trough around the obstacle with a fine diamond bur. The tip of an ultrasonic unit is then applied to the side of the post fragment at full power with water irrigation. Ultrasonic vibration is applied for periods of a few seconds followed by drying with compressed air. This should lead to dislodgement of the fragment of the post, which can then be removed with a fine forceps. When an obstruction allows for limited access to the coronal portion of the point, a more conservative approach would be to try to consume it instead of consuming the surrounding dentin. An important point to realize when removing silver points is that one is dealing with a very soft material. Any misdirection of a bur can sever the point, complicating the case even further. US has proven to be very helpful in the removal of these points. Simply trough around the silver point with an ultrasonic spreader tip and carefully eliminate dentin while following the long axis, taking care not to cut the point. The space created around the silver point will usually loosen the silver point, which can then be removed with a Steiglitz forceps or hemostat. At all times, the use of intraoral radiographs is recommended to confirm the position and the remaining length of the obstruction, as well as the thickness of canal walls. The time required for removal of a post or silver point is influenced by the nature of the obstruction, its diameter, and location.

\section{Increased Action Of Irrigating Solutions}

The effectiveness of irrigation relies on both the mechanical flushing action and the chemical ability of irrigants to dissolve tissue [8]. The flushing action from syringe irrigation is relatively weak and dependent not only on the anatomy of the root canal but also on the depth of placement and the diameter of the needle. It has been shown that irrigants can only progress $1 \mathrm{~mm}$ beyond the tip of the needle.

The only effective way to clean webs and fins is through movement of the irrigation solution, as they cannot be mechanically cleaned. US is a useful adjunct in cleaning these difficult anatomical features. It has been demonstrated that an irrigant in conjunction with ultrasonic vibration, which generates a continuous movement of the irrigant, is directly associated with the effectiveness of the cleaning of the root canal space.

Acoustic streaming, as described by Ahmad et al., has been shown to produce sufficient shear forces to dislodge debris in instrumented canals. When files were activated with ultrasonic energy in a passive manner, acoustic streaming was sufficient to produce significantly cleaner canals compared with hand filing alone. Similarly, Jensen et al. recommended a vibrating file of small size subjected to a high power setting, as smaller files will be less likely to contact the canal walls.

\section{Ultrasonic Condensation Of Gutta-Percha}

Ultrasonically activated spreaders have been used to thermoplasticize gutta-percha in a warm lateral condensation technique [9]. In some in vitro experiments, this was demonstrated to be superior to conventional lateral condensation with respect to sealing properties and density of gutta-percha. Ultrasonic spreaders that vibrate linearly and produce heat, thus thermoplasticizing the gutta-percha, achieved a more homogeneous mass with a decrease in number and size of voids and produced a more complete three-dimensional obturation of the root canal system. This technique has also been evaluated clinically with favorable results. A number of obturation protocols have been described for ultrasonic condensation of gutta-percha: (a) ultrasonic softening of the master cone followed by cold lateral condensation; (b) one or two times of ultrasonic activation after completion of cold lateral condensation; (c) ultrasonic activation after placement of each second accessory cone; or (d) ultrasonic activation after placement of each accessory cone.

The obturation technique recommended when using the ultrasonic techniques consists of initial placement of a gutta-percha cone to the working length followed by cold lateral condensation of two or three accessory cones using a finger spreader. The ultrasonic spreader is then placed into the center of the guttapercha mass $1 \mathrm{~mm}$ short of the working length and activated at intermediate power to prevent charring of root surfaces and fracture of the ultrasonic 
spreader. After activation, the ultrasonic spreader is removed, and an additional accessory cone is placed, followed by energizing with the activated ultrasonic spreader. This process is repeated until the canal is filled. During each subsequent step, the ultrasonic spreader should be placed slightly more coronally. The ultrasonic spreader must be in the mass of gutta-percha for about 10 seconds to achieve thermoplasticization. Leaving it in the canal for more than 10 seconds can produce a rise in temperature that is damaging to the root surface.

In addition, it has been demonstrated that placement of sealers with an ultrasonically energized file promoted a better covering of canal walls with better filled accessory canals (evaluated by radiography) than placement of sealers with hand instruments.

\section{Placement Of Mineral Trioxide Aggregate (MTA)}

Witherspoon and Ham [10] described the use of US to aid in the placement of MTA. The inherent irregularities and divergent nature of some open apices may predispose the material to marginal gaps at the dentin interface. It was demonstrated that, with the adjunct of US, a significantly better seal with MTA was achieved. Placement of MTA with ultrasonic vibration and an endodontic condenser improved the flow, settling, and compaction of MTA. Furthermore, the ultrasonically condensed MTA appeared denser radiographically, with fewer voids. The recommended placement method consists of selecting a condenser tip, then picking up and placing the MTA with the ultrasonic tip, followed by activating the tip and slowly moving the MTA material down using a 1- to 2-mm vertical packing motion. Direct ultrasonic energy will vibrate and generate a wavelike motion, which facilitates moving and adapting the cement to the canal walls. In a case of repairing a defect apical to the canal curvature, Ruddle recommends incrementally placing MTA deep into a canal, then shepherding it around the curvature with a flexible trimmed gutta-percha cone utilized as a plugger. A precurved 15 or 20 stainless steel file is then inserted into the material and placed to within 1 or $2 \mathrm{~mm}$ of the working length. This is followed by indirect ultrasound, which involves placing the working end of an ultrasonic instrument on the shaft of the file. This vibratory energy encourages MTA to move and conform to the configurations of the canal laterally as well as controlling its movement. This technique was recommended initially for placing MTA in open and diverging apices, but it can also be used to put the material in root-end cavities, in perforations, and especially in perforations of the floor of the pulp chamber.

\section{VII.Surgical Endodontics: Root-End Cavity Preparation And Refinement And Placement Of Root- End Obturation Material}

Since sonically or ultrasonically driven microsurgical retrotips became commercially available in the early 1990s, this new technique of retrograde root canal instrumentation has been established as an essential adjunct in periradicular surgery [11]. The development of ultrasonic and sonic retrotips has revolutionized root end therapy, improving the surgical procedure with better access to the root end, resulting in better canal preparation. Ultrasonic retrotips come in a variety of shapes and angles, thus improving some steps during the surgical procedures.

At first glance, the most relevant clinical advantages are the enhanced access to root ends in a limited working space. This leads to a smaller osteotomy for surgical access because of the advantage of using various angulations and the small size of the retrotips. It also have additional advantages, such as deeper and more conservative cavities that follow the original path of the root canal more closely. A better-centered root-end preparation also lessens the risk of lateral perforation. Furthermore, the geometry of the retrotip design does not require a beveled root-end resection for surgical access, thus decreasing the number of exposed dentinal tubules and minimizing apical leakage.

They also enable the removal of isthmus tissue present between two canals within the same root. It is considered a time saving technique that seems to have a lower failure rate. Furthermore, US produced less smear layer in a retro-end cavity compared to a slow-speed handpiece.

A condenser tip ultrasonically activated can be utilized for placement of retrograde filling materials, as the ultrasonic vibration is meant to improve the flow, settling and compaction of these materials to rootend dentinal walls. This should improve the delivery of materials into the cavity thus enhancing their seal.

Ultrasonic tips can also be used to polish root end material and apical surfaces. Utilizing specific ultrasonic tips for refinement of the external radicular surface may be beneficial in the elimination of extraradicular bacteria, which may be responsible for infection.

\section{Root Canal Preparation}

Ultrasonic devices were introduced for use in root canal preparation in 1957 by Richman [1]. Several studies have shown that ultrasonically or sonically prepared teeth have significantly cleaner canals than teeth prepared by hand instruments [12]. Numerous studies have analyzed the different characteristics of 
ultrasonically activated files, such as cutting efficiency, effect on bacteria, characteristics of root canal preparation, mechanical and technical features of files and handpieces, and clinical implications.

\section{Conclusion}

It can be concluded from this review of the literature that US offers many applications and advantages in clinical endodontics. At many instances it has proved as a successful adjunct to various conventional techniques. Finally, integration of US that is Endosonics has changed the way endodontics is being practiced today.

\section{References}

[1]. Richman RJ. The use of ultrasonics in root canal therapy and root resection. Med Dent J, 12, 1957,12-8.

[2]. Martin H, Cunningham W. Endosonic endodontics: the ultrasonic synergistic system. Int Dent J,34, 1984,198 -203.

[3]. Martin H, Cunningham W. Endosonics: the ultrasonic synergistic system of endodontics. Endod Dent Traumatol 1985;1:201-6.

[4]. Stock CJR. Current status of the use of ultrasound in endodontics. Int Dent J, 41 1991, 175-82.

[5]. Sempira HN, Hartwell GR. Frequency of second mesiobuccal canals in maxillary molars as determined by use of an operating microscope: a clinical study. J Endod, 26, 2000, 673-4.

[6]. Ward JR, Parashos P, Messer HH. Evaluation of an ultrasonic technique to remove fractured rotary nickel-titanium endodontic instruments from root canals: an experimental study. J Endod, 29, 2003, 756-63.

Buoncristiani J, Seto BG, Caputo AA. Evaluation of ultrasonic and sonic instruments for intraradicular post removal. J Endod, 20,1994,486-9.

[7]. Abou-Rass M, Piccinino MV. The effectiveness of four clinical irrigation methods on the removal of root canal debris. Oral Surg Oral Med Oral Pathol, 54, 1982, 323-8.

[8]. Moreno A. Thermomechanical softened gutta-percha root canal filling. J Endod, 3, 1977, 186-8.

[9]. Witherspoon D, Ham K. One-visit apexification: technique for inducing root-end barrier formation in apical closures. Pract Proced Aesthet Dent, 13, 2001, 455-60.

[10]. Sumi Y, Hattori H, Hayashi K, Ueda M. Ultrasonic root-end preparation: clinical and radiographic evaluation of results. J Oral Maxillofac Surg, 54, 1996, 590-3.

[11]. Tronstad L, Barnett F, Schawartzben L, Frasca P. Effectiveness and safety of a sonic vibratory endodontic instrument. Endod Dent Traumatol, 1, 1985, 69-76. 